\title{
Current Progress of Aptamer-Based Molecular Imaging
}

\author{
Andrew Z. Wang ${ }^{1}$, and Omid C. Farokhzad ${ }^{2,3}$ \\ ${ }^{1}$ Department of Radiation Oncology, Lineberger Comprehensive Cancer Center, Carolina Center for Cancer Nanotechnology \\ Excellence, University of North Carolina-Chapel Hill, Chapel Hill, North Carolina; ${ }^{2}$ Laboratory of Nanomedicine and Biomaterials, \\ Department of Anesthesiology, Brigham and Women's Hospital, Harvard Medical School, Boston, Massachusetts; and ${ }^{3}$ King \\ Abdulaziz University, Jeddah, Saudi Arabia
}

\begin{abstract}
Aptamers, single-stranded oligonucleotides, are an important class of molecular targeting ligand. Since their discovery, aptamers have been rapidly translated into clinical practice. They have been approved as therapeutics and molecular diagnostics. Aptamers also possess several properties that make them uniquely suited to molecular imaging. This review aims to provide an overview of aptamers' advantages as targeting ligands and their application in molecular imaging.
\end{abstract}

Key Words: aptamers; molecular imaging probes; molecular diagnostics; aptamer

J Nucl Med 2014; 55:353-356

DOI: 10.2967/jnumed.113.126144

Molecular imaging uses specific probes, rather than the nonspecific contrast agents used in conventional imaging, to visualize a particular target or biologic process. These imaging probes function either by targeting a physiologic process or by targeting cellular biomarkers. The latter type of imaging probe requires biologic targeting ligands that can bind to cellular biomarkers with high sensitivity, specificity, and affinity. Although the development of such biologic targeting ligands has focused mainly on peptides and antibodies, aptamers are a relatively new type of targeting ligand and hold several key advantages over their protein counterparts. This article aims to review the advantages of aptamers as targeting ligands and the current progress in using aptamers in molecular imaging.

\section{APTAMERS AS MOLECULAR TARGETING LIGANDS}

Aptamers are single-stranded DNA or RNA oligonucleotides that are approximately $20-100$ bases in length (1). These molecules can spontaneously fold into well-defined 3-dimensional structures and bind to their target molecules with high affinity and specificity (Fig. 1). Aptamers can be

Received Nov. 20, 2013; revision accepted Jan. 21, 2014.

For correspondence or reprints contact: Andrew Z. Wang, University of North Carolina-Chapel Hill, 101 Manning Dr., CB 7512, Chapel Hill, NC 27599-7512.

E-mail: zawang@med.unc.edu

Published online Feb. 13, 2014.

COPYRIGHT (c) 2014 by the Society of Nuclear Medicine and Molecular Imaging, Inc. selected to recognize a wide range of molecules, including proteins, phospholipids, sugars, and other nucleic acids. The selection method is called systemic evolution of ligands by exponential enrichment (SELEX), which was first described in 1990 (2). In SELEX, a large library $\left(>10^{10}\right)$ of random sequences of nucleic acids is subjected to a selection process against a particular target. The aptamers that can bind to the target molecule more tightly are preferentially amplified by each round of selection. After rounds of selection, the aptamer that binds the target molecule with the highest affinity and specificity is isolated.

Although aptamers are similar to antibodies in their ability to recognize and bind to target molecules, they hold several advantages over antibodies as targeting ligands (3). First, aptamers fold into 3-dimensional structures spontaneously, making them more resistant than antibodies to $\mathrm{pH}$ and temperature changes. Antibodies, in contrast, generally cannot regain their original conformation once they have been denatured. Such high stability also allows aptamers to be more easily modified through chemical means than are antibodies. Second, unlike antibodies, which require in vitro or in vivo production, aptamers are synthesized chemically. Chemical synthesis of a short nucleic acid strand is more reliable, and there is much less variation between production batches. Furthermore, aptamers are more economical to produce and the scale-up is easier for aptamers than for antibodies. Another important advantage of aptamers is that the selection process is not dependent on the immunogenicity or the toxicity of the target. Lastly, aptamers are much smaller than antibodies. Their small size can improve tissue penetration and increase systemic clearance; both are excellent properties for imaging probes.

Aptamers also have some disadvantages and challenges. As single-strand nucleic acids, they are prone to degradation by nucleases. Therefore, aptamers require chemical modifications to slow down the degradation process (3). Although their small size is an advantage, it can also be a disadvantage because aptamers' clearance may be too rapid. The fast clearance has largely limited aptamers' translation as therapeutic agents.

\section{CLINICAL APPLICATIONS OF APTAMERS}

Since their discovery, nucleic acid aptamers have been developed for various clinical applications, including in vitro 


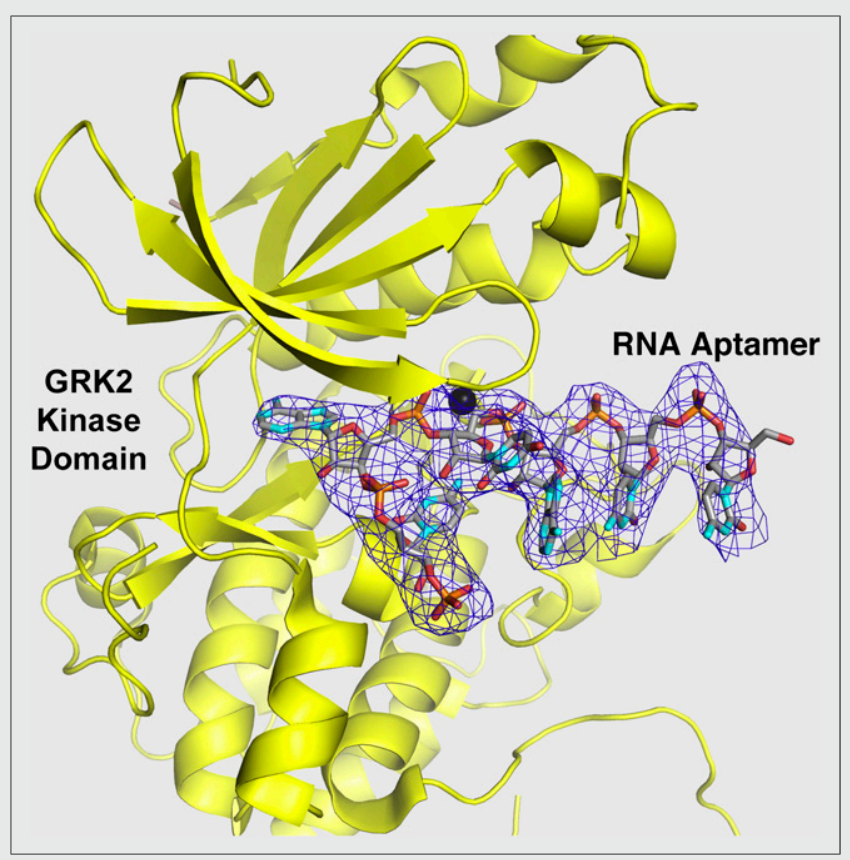

FIGURE 1. Binding of ${ }^{13} \mathrm{C}-\mathrm{RNA}$ aptamer to $\mathrm{G}$ protein-coupled receptor kinase (GRK2). (Reprinted with permission of (20).)

diagnostics, molecular imaging, biomarker discovery, and therapeutics. Current in vitro diagnostics, such as enzymelinked immunosorbent assays, rely primarily on antibody technology. As mentioned above, aptamers are able to target molecules that are difficult for antibody generation. For this reason and because of aptamers' lower production cost, there is growing interest in the development of aptamer-based in vitro diagnostics. Indeed, aptamer diagnostics have been developed for the detection of a wide range of molecules (4). The first successfully commercialized aptamer-based diagnostic assay is one that detects mycotoxin in grains for agriculture applications (5). Aptamers have also been shown to enable large-scale proteomic studies, which can also be used for the discovery of novel biomarkers (6). Another key focus in the clinical translation of aptamers has been the development of aptamer-based therapeutics. Therapeutic strategies generally involve binding of an aptamer directly to a therapeutic target to modulate its downstream signaling or to deliver a therapeutic cargo. Because aptamers undergo rapid systemic clearance, aptamer therapeutics are best suited to applications in which systemic circulation is avoided (e.g., ocular delivery) or the disease process is temporal, such as clotting and bleeding. Today, there is one Food and Drug Administration-approved aptamer therapeutic, pegaptanib (Macugen; Valeant Pharmaceuticals), with many more under clinical investigation (7). Pegaptanib is a pegylated aptamer that targets vascular endothelial growth factor, which plays a critical role in angiogenesis involved in age-related macular degeneration. Lastly, aptamers have also been studied for applications in molecular imaging. These applications will be reviewed in detail in the following sections.

\section{OPTIC IMAGING WITH APTAMER-BASED PROBES}

Optic imaging, which includes fluorescence and bioluminescence, is a key imaging modality for visualization of molecular processes both on the cellular level and on the organismic level in small animals. Optical molecular imaging is critical in understanding physiologic and disease processes and can provide invaluable insight to disease treatment. Aptamers' small size and ability to target a wide range of molecules make them excellent molecular imaging probes for cellular imaging. Aptamer-based imaging probes can be formulated either by directly linking them to fluorescent molecules or by conjugating aptamers to nanoparticlebased optical probes (8). These aptamer-based molecular imaging probes have been used to image biomarkers and cellular proteins such as integrins, prostate-specific membrane antigen (PSMA), and nucleolin. Though similar to antibody-based probes, the small size of aptamers makes them better suited to the imaging of intracellular proteins than antibodies.

Aptamers have several unique properties, which have also enabled novel imaging applications. Shi et al. used the conformation change associated with aptamer-target binding to generate activatable aptamer probes (AAP) (9). In this study, the authors engineered an AAP, sgc8 (41b, dissociation constant $\left[\mathrm{K}_{\mathrm{d}}\right]=0.8 \mathrm{nM}$ ), that binds to CCRF-CEM lymphoblastic lymphoma cells. Sgc8 is made up of 3 components: an aptamer sequence that binds to CCRF-CEM cells, a linker, and a short DNA sequence with both a fluorophore and a quencher covalently attached at either terminus. When the aptamer is free, its conformation keeps the fluorophore near the quencher, resulting in quenched fluorescence. However, when the probe binds to a target, its conformation change separates the fluorophore from the quencher, resulting in active fluorescence. The authors demonstrated that AAPs can be used in in vivo molecular imaging in small animals.

Aptamers' ability to target a wide range of biomarkers, including small molecules, has allowed unique applications for aptamer molecular probes. For example, Wu et al. developed aptamers that can bind to adenosine triphosphate, a small-molecule target with a significant role in cellular functions and signaling (10). Moreover, they integrated the AAP concept from above, enabling the imaging and detection of cellular adenosine triphosphate changes in real time.

\section{MR IMAGING}

Although MR imaging is generally known as an anatomic imaging modality, it can be used in molecular imaging with the proper imaging probes. Aptamers have been conjugated to paramagnetic molecules, including gadolinium-containing compounds and superparamagnetic iron oxide nanoparticles (SPIONs), for MR imaging-based molecular imaging (7). Our group has conjugated the A10 aptamer $\left(56 \mathrm{~b}, \mathrm{~K}_{\mathrm{d}}=20.5\right.$ $\mathrm{nM}$ ), targeted against PSMA on prostate cancer cells, to SPIONs (11). We demonstrated that the A10-SPION conjugate can 
target PSMA-expressing prostate cancer cells with high sensitivity and specificity and can be used as an MR imaging agent. Moreover, using the ability of aptamers to carry doxorubicin, a chemotherapeutic, we showed that we can also deliver doxorubicin to prostate cancer in a targeted fashion. In another study, Yigit el al. conjugated 2 aptamers that can target thrombin to SPIONS (12). The 2 aptamers, Thrm-A and Thrm-B, target the fibrinogen-recognition exosite and the heparin binding exosite of thrombin, respectively. The investigators showed that aptamer-functionalized SPIONs can be used to detect thrombin. As a method to improve specificity, the best MR signal is generated when thrombin binds to both Thrm-A and Thrm-B SPION conjugates.

\section{CT IMAGING}

Aptamer-based molecular probes have also been used in CT imaging. Kim et al. conjugated the A10 aptamer, targeted against PSMA, to gold nanoparticles (13). Gold nanoparticles, because of their high atomic number, can act as CT imaging contrast agents. The investigators showed that A10-targeted nanoparticles can bind to PSMA-expressing prostate cancer cells with high sensitivity and specificity. More importantly, they showed that the A10-gold nanoparticle conjugate can be used as a molecular probe for the detection of PSMAexpressing cancer cells by CT imaging.

\section{ULTRASOUND IMAGING}

Ultrasound is one of the most commonly used clinical imaging modalities. One of its main limitations is image resolution. To overcome this limitation, contrast agents, such as microbubbles, have been developed (14). To enable molecular imaging, investigators have conjugated aptamers to microbubbles to formulate molecular ultrasound probes (15). Nakatsuka et al. engineered a novel aptamer-cross-linked microbubble as a molecular ultrasound imaging agent (16). The microbubbles are designed to show ultrasound activation only at levels of thrombin associated with clot formation. To accomplish this, the microbubbles are coated with polymer-DNA strands and aptamers that can bind to thrombin. The aptamers also contain sequences that can bind to the DNA in a polymer-DNA complex, enabling cross-linking on the microbubble surface. However, the binding of thrombin to aptamer will displace the aptamers from the polymer-DNA complex. The investigators demonstrated that these microbubbles can act as a stimulus-responsive contrast agent and generate ultrasound signal in response to only the levels of thrombin. Such an aptamer-based ultrasound contrast agent can be useful for imaging thrombosis.

\section{NUCLEAR IMAGING WITH APTAMER-BASED PROBES}

Nuclear imaging holds the highest potential for the clinical translation of aptamer-based molecular imaging probes. The very first report of an aptamer-based imaging probe was a radiolabeled $\left({ }^{99 \mathrm{~m}} \mathrm{Tc}\right)$ aptamer targeted against human neutrophil elastase to identify sites of inflammation (8). When compared with a radiolabeled $\mathrm{IgG}$, the aptamer probe achieved a higher target-to-background ratio (4-fold) than that of $\operatorname{IgG}$ (3-fold). This difference was attributed to the more rapid clearance of unbound aptamer probes than $\mathrm{IgG}$ probes.

The study on radiolabeled TTA1 best demonstrated the potential of aptamers as nuclear imaging probes (17). The investigators radiolabeled the aptamer TTA $1\left(38 \mathrm{~b}, \mathrm{~K}_{\mathrm{d}}=5\right.$ $\mathrm{nM})$ with ${ }^{99 \mathrm{~m}} \mathrm{Tc}$. TTA1 is targeted against the extracellular matrix protein tenascin- $\mathrm{C}$, which is expressed during tissue remodeling processes including angiogenesis and tumor growth. Tenascin-C is also known to be overexpressed by a wide range of tumors, including lung, breast, and prostate. Radiolabeled TTA1 was evaluated in mouse xenograft models of brain and breast cancer. The investigators showed that TTA1 has a rapid clearance profile, with a blood half-life of 2 min. The aptamers penetrated tumors quickly, with $6 \%$ of injected dose in tumor at $10 \mathrm{~min}$. At $60 \mathrm{~min}$ after injection, there is still $3 \%$ of the injected dose in tumor. The tumor-toblood ratio was 50 within $3 \mathrm{~h}$, highlighting its high potential for clinical translation (Fig. 2). Despite these exciting data, TTA1 has not been translated clinically. One main reason can be the unclear significance of tenascin in clinical oncology. Recently, Pieve et al. has formulated ${ }^{99 \mathrm{~m}} \mathrm{Tc}$-labeled aptamer probes that are targeted against the MUC1 protein, a well-established biomarker for several tumors, including breast cancer (18). Similar to previous studies, these aptamer probes have rapid clearance and can target MUC1-expressing cells in vivo in a mouse xenograft model of cancer.

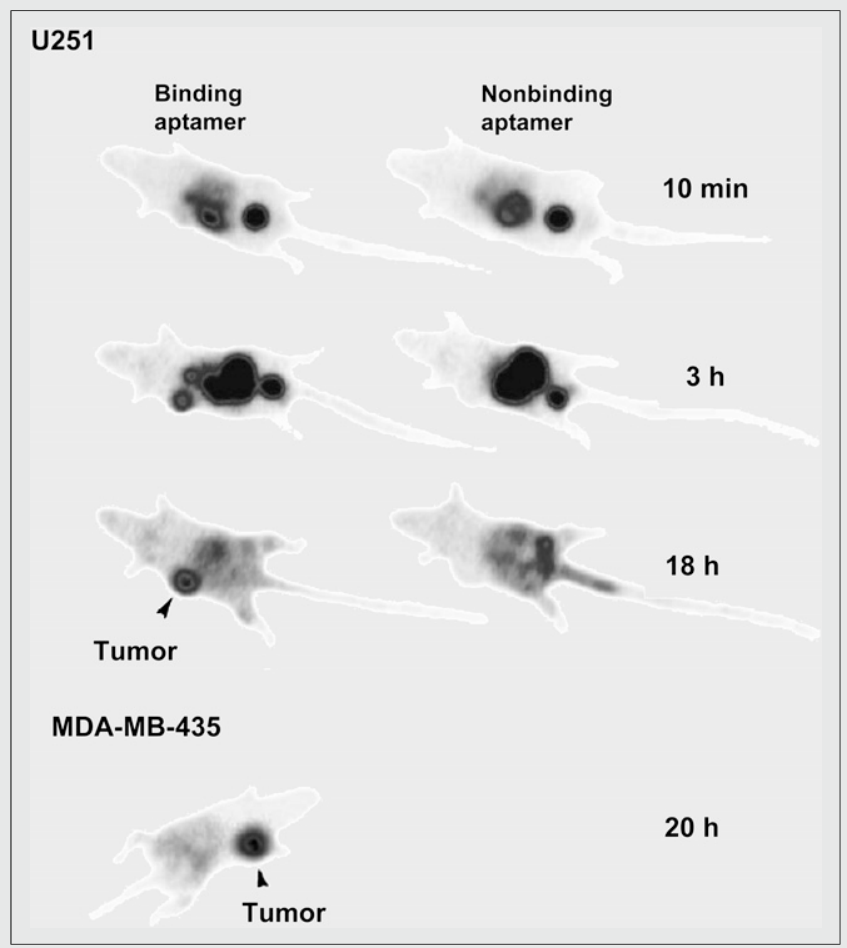

FIGURE 2. Nuclear imaging with radiolabeled TTA1. Molecular probe is able to detect both U251 glioblastoma xenograft and MDA-MB-235 breast tumor xenograft. 
Radiolabeled aptamers have also been used in cellular imaging and biologic studies. Hwang et al. conjugated the AS1411 aptamer, targeted against nucleolin, to a cobalt-ferrite nanoparticle that is also coated with fluorescent rhodamine (19). The conjugate was then labeled with ${ }^{67} \mathrm{Ga}$. The final probe can be detected by fluorescent imaging, MR imaging, and nuclear imaging. They demonstrated that the AS1411nanoparticle probe can bind to nucleolin-expressing tumor cells with specificity in vitro and in vivo.

\section{CONCLUSION AND FUTURE DIRECTIONS}

Aptamers, a relatively new class of targeting ligand, have already made a significant impact on clinical medicine. Current research and clinical translation efforts are focused on developing aptamers as therapeutics and molecular diagnostics. These efforts have resulted in one therapeutic that is Food and Drug Administration-approved, as well as several more therapeutics and diagnostics under investigation. On the other hand, aptamers also possess many unique properties that are well suited to molecular imaging. Because of these properties, aptamers are studied in a wide range of applications in molecular imaging. There continues to be high interest in developing aptamer-based probes in preclinical research. These studies will improve the technology and identify the potential applications for aptamers in molecular imaging. The successful clinical translation of aptamerbased molecular imaging probes will also require the development of aptamers against biomarkers that have high clinical significance. In summary, aptamers are excellent molecular targeting ligands, and they hold great promise in improving molecular imaging.

\section{DISCLOSURE}

This work was supported by the University Cancer Research Fund from the University of North Carolina (AZW), R01CA178748-01 from the National Institutes of Health/ National Cancer Institute (AZW), U54-CA151652 from the NIH Center for Nanotechnology Excellence (AZW), U54CA151884 from the National Cancer Institute Center of Cancer Nanotechnology Excellence at MIT-Harvard (OCF), HHSN268201000045C from the National Heart, Lung, and Blood Institute Programs of Excellence in Nanotechnology
(OCF), and the Koch-Prostate Cancer Foundation Award in Nanotherapeutics (OCF). No other potential conflict of interest relevant to this article was reported.

\section{REFERENCES}

1. Kong RM, Zhang XB, Chen Z, Tan W. Aptamer-assembled nanomaterials for biosensing and biomedical applications. Small. 2011;7:2428-2436.

2. Ellington AD, Szostak JW. In vitro selection of RNA molecules that bind specific ligands. Nature. 1990;346:818-822.

3. Hu M, Zhang K. The application of aptamers in cancer research: an up-to-date review. Future Oncol. 2013;9:369-376.

4. Hong P, Li W, Li J. Applications of aptasensors in clinical diagnostics. Sensors (Basel). 2012;12:1181-1193.

5. Cruz-Aguado JA, Penner G. Fluorescence polarization based displacement assay for the determination of small molecules with aptamers. Anal Chem. 2008;80: 8853-8855.

6. Kraemer S, Vaught JD, Bock C, et al. From SOMAmer-based biomarker discovery to diagnostic and clinical applications: a SOMAmer-based, streamlined multiplex proteomic assay. PLOS ONE. 2011;6:e26332.

7. Ni X, Castanares M, Mukherjee A, Lupold SE. Nucleic acid aptamers: clinical applications and promising new horizons. Curr Med Chem. 2011;18:4206-4214.

8. Hong H, Goel S, Zhang Y, Cai W. Molecular imaging with nucleic acid aptamers. Curr Med Chem. 2011;18:4195-4205.

9. Shi H, He X, Wang K, et al. Activatable aptamer probe for contrast-enhanced in vivo cancer imaging based on cell membrane protein-triggered conformation alteration. Proc Natl Acad Sci USA. 2011;108:3900-3905.

10. Wu C, Chen T, Han D, et al. Engineering of switchable aptamer micelle flares for molecular imaging in living cells. ACS Nano. 2013;7:5724-5731.

11. Wang AZ, Bagalkot V, Vasilliou CC, et al. Superparamagnetic iron oxide nanoparticle-aptamer bioconjugates for combined prostate cancer imaging and therapy. ChemMedChem. 2008;3:1311-1315.

12. Yigit MV, Mazumdar D, Lu Y. MRI detection of thrombin with aptamer functionalized superparamagnetic iron oxide nanoparticles. Bioconjug Chem. 2008;19: 412-417.

13. Kim D, Jeong YY, Jon S. A drug-loaded aptamer-gold nanoparticle bioconjugate for combined CT imaging and therapy of prostate cancer. ACS Nano. 2010;4:3689-3696.

14. Unnikrishnan S, Klibanov AL. Microbubbles as ultrasound contrast agents for molecular imaging: preparation and application. AJR. 2012;199:292-299.

15. Wang CH, Huang YF, Yeh CK. Aptamer-conjugated nanobubbles for targeted ultrasound molecular imaging. Langmuir. 2011;27:6971-6976.

16. Nakatsuka MA, Mattrey RF, Esener SC, Cha JN, Goodwin AP. Aptamercrosslinked microbubbles: smart contrast agents for thrombin-activated ultrasound imaging. Adv Mater. 2012;24:6010-6016.

17. Winnard PT Jr, Pathak AP, Dhara S, Cho SY, Raman V, Pomper MG. Molecular imaging of metastatic potential. J Nucl Med. 2008;49(suppl 2):96S-112S.

18. Pieve CD, Perkins AC, Missailidis S. Anti-MUC1 aptamers: radiolabelling with ${ }^{99 \mathrm{~m} T \mathrm{Tc}}$ and biodistribution in MCF-7 tumour-bearing mice. Nucl Med Biol. 2009;36:703-710.

19. Hwang do W, Ko HY, Lee JH, et al. A nucleolin-targeted multimodal nanoparticle imaging probe for tracking cancer cells using an aptamer. $J$ Nucl Med. 2010;51: 98-105.

20. Tesmer VM, Lennarz S, Mayer G, Tesmer JJ. Molecular mechanism for inhibition of g protein-coupled receptor kinase 2 by a selective RNA aptamer. Structure. 2012;20:1300-1309. 